\title{
Extensive and recalcitrant common warts in an immunocompetent patient: rapid and complete remission after oral isotretinoin monotherapy
}

\author{
Efstathia Pasmatzi ${ }^{1 凶}$, George Badavanis ${ }^{2}$, Nikiforos Kapranos 3 , Assimina Apostolidou², Alexandra Monastirli ${ }^{1,2}$, \\ Dionysios Tsambaos ${ }^{1,2}$
}

${ }^{1}$ Department of Dermatology, University of Patras, Rio-Patras, Greece. ${ }^{2}$ Center for Dermatologic Diseases, Limassol, Cyprus. ${ }^{3}$ Laboratory for Molecular Histopathology, Athens, Greece.

\begin{abstract}
An immunocompetent patient with extensive and recalcitrant common warts that was orally treated with isotretinoin ( $1 \mathrm{mg} / \mathrm{kg} /$ day) is reported. His lesions revealed a complete remission after 6 weeks of treatment, which was well tolerated. The patient has presently completed a 23-month follow-up and shows no evidence of relapse of his skin lesions. In view of these remarkable therapeutic results, further randomized controlled clinical studies in large numbers of patients are now warranted, which will definitely determine whether monotherapy with oral isotretinoin at a dose of $1 \mathrm{mg} / \mathrm{kg} /$ day may be regarded as a highly effective and welltolerated therapeutic modality for extensive and recalcitrant common warts in both immunocompetent and immunocompromised patients.
\end{abstract}

Keywords: retinoids, isotretinoin, warts, human papilloma virus

Received: 14 January 2020 | Returned for modification: 12 February 2020 | Accepted: 14 February 2020

\section{Introduction}

Common warts are benign, small, mucocutaneous grainy lesions that are caused by human papilloma virus (HPV; particularly HPV types 1, 2, 4, 27, 29, and 57), mostly spread by direct contact or autoinoculation. They can arise on any part of the body, but they more frequently occur on the hands, fingers, feet, and knees. Although common warts are generally asymptomatic, they may become painful upon application of pressure, particularly when they develop on the palms and soles (1).

Oral isotretinoin (13-cis retinoic acid), a synthetic compound of the first retinoid generation, is the drug of choice for the treatment of severe and therapy-resistant forms of acne that are unresponsive to conventional therapy, including systemic antibiotics. Moreover, this retinoid has been successfully applied in the systemic treatment of a wide spectrum of benign and malignant cutaneous disorders (2). Here we report the rapid and complete remission of extensive and recalcitrant common warts in an immunocompetent patient after oral isotretinoin monotherapy.

\section{Case report}

A 26-year-old HIV-negative and otherwise healthy Caucasian man presented with a 30-month-history of multiple warty skin lesions on both upper extremities. They had previously been treated by other dermatologists with laser, cryotherapy, and topical trichloroacetic acid, but each time they relapsed shortly after completion of therapy and became more extensive. His past medical history was unremarkable. In particular, he had no diabetes mellitus, recurrent infections, or recreational drug use/abuse. His family history was also unremarkable.

Physical examination revealed multiple and focally confluent, hyperkeratotic, whitish papules with an irregular verrucous sur- face that were localized on the extensor surface of both underarms (Fig. 1A). The results of hematological, biochemical, and serological examinations, including quantitative determination of lymphocyte subpopulations and of serum immunoglobulins, serum protein immunoprecipitation, and autoimmune (antinuclear, anti-ds-DNA, anti-SM, anti-RNP, anti-SSA, anti-SSB antibodies) and tumor markers (a-fetoprotein, carcinoembryonic antigen, Ca 19-9, prostate-specific antigen, and beta-2-microglobulin), were either negative or within normal limits. The results of chest X-ray and ultrasound examinations of the abdomen and pelvis were unremarkable. Histological examination of a skin biopsy revealed typical features of common warts; for example, acanthosis, papillomatosis, hyperkeratosis, compact orthokeratosis with focal parakeratosis overlying the papillary tops, hypergranulosis with keratohyalin condensation, and perinuclear vacuolization.

Considering the recalcitrance of the lesions of our patient and in view of the previously reported impressive therapeutic efficacy of oral isotretinoin in an immunosuppressed patient with extensive and recalcitrant common warts (3), the decision was made to introduce oral treatment with isotretinoin $(1 \mathrm{mg} / \mathrm{kg} /$ day; Rotaxin, Hadjiconstantinou Bros. Ltd., Limassol, Cyprus). Because our patient was 26 years old, age limitations (isotretinoin is not indicated for treatment in children $<12$ years) were not applicable. Written consent was obtained from the patient subsequent to a thorough explanation of the possible therapeutic efficacy and the known side effects of this retinoid.

Already after 2 weeks of continuous oral isotretinoin treatment, a considerable reduction in the number and size of common warts could be seen. Complete remission was observed after 6 weeks of oral administration of this retinoid (Fig. 1B). The patient developed cheilitis and dryness of the nasal mucosa with occasional epistaxis, whereas his laboratory parameters revealed no significant alteration. He has presently completed a 23-month follow-up 
and shows no evidence of relapse of his skin lesions.

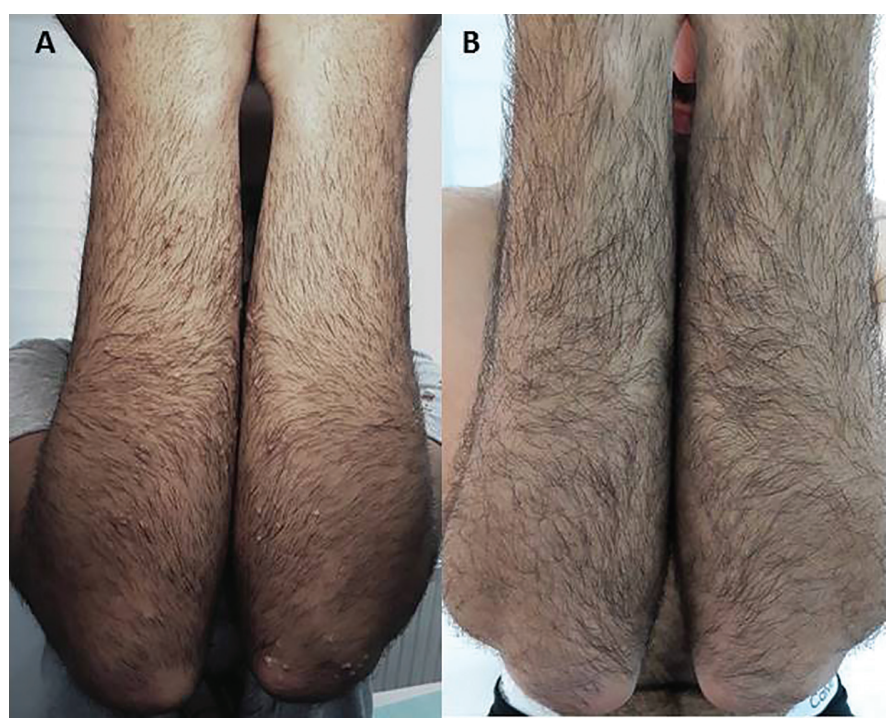

Figure 1 | Clinical aspect of the extensive common warts on the extensor surface of both underarms (A) before and (B) 6 weeks after onset of oral isotretinoin monotherapy. There is complete remission of common warts, whereas in some areas isotretinoin-induced discrete fine scales can be seen.

\section{Discussion}

In most immunocompetent patients, spontaneous remission of common warts is possible, but it often takes a long time, even years, to occur (4). Moreover, apart from their tendency to spread, common warts cause significant disfigurement and have a negative impact on the patient's quality of life; thus, rapid and effective therapeutic intervention is warranted in most cases (5). Diverse therapeutic approaches include electrocautery, laser ablation, and cryosurgery; topical applications of caustic and antimitotic drugs or immune modulators have been used in the management of common warts, but they are often associated with an unsatisfactory response, high recurrence rates, and side effects, such as severe topical reactions, temporary or permanent discoloration, and scarring. Common warts often represent a frustrating therapeutic problem, particularly in immunocompromised patients, because despite treatment they exhibit a progressive increase in their size and area of skin involvement, and they also have the potential for malignant transformation into squamous cell carcinomas (3).

Data on the treatment of common warts with oral isotretinoin are sparse. Katz (6) was the first to report the introduction of this compound for the systemic treatment of common warts. About 1 year after completion of chemotherapy for Hodgkin's disease, his immunosuppressed patient developed large common warts on the hands and feet, which were refractory to all conventional forms of therapy. Initial oral administration of $1 \mathrm{mg} / \mathrm{kg} /$ day of isotretinoin over a period of 10 weeks led to a considerable regression of the wart on his right foot, but it had limited influence on lesions located elsewhere. After 28 weeks of treatment, the isotretinoin dosage was decreased to $0.5 \mathrm{mg} / \mathrm{kg} /$ day; nevertheless, the patient's foot wart continued to improve, but it grew larger upon further reduction of the dosage. Re-administration of $0.5 \mathrm{mg} / \mathrm{kg} /$ day was reportedly accompanied by clinical improvement of the wart but was unable to lead to complete remission of the lesions.

In 2005 our group was the first to report the complete remission of extensive and recalcitrant common warts after 10 weeks of oral isotretinoin therapy at a dose of $1 \mathrm{mg} / \mathrm{kg} /$ day in an immunocompromised patient (low-grade B-cell lymphoma), who remained free of any evidence of relapse of his cutaneous lesions during the 24-month follow-up period (3).

Dave and Abdelmaksoud (7) reported complete disappearance of the lesions in 12 patients with common warts under oral treatment with low-dose isotretinoin (10 to $20 \mathrm{mg}$ every other day); however, no definite conclusions can be drawn from their study with regard to the therapeutic efficacy of this compound because, apart from the oral administration of isotretinoin, "infrequent topical application of 5-FU, imiquimod, salicylic or lactic acid, and electrocautery was permitted.”

More recently, Herold et al. (8) reported successful treatment of recalcitrant common warts in two solid organ transplant recipients with low-dose oral isotretinoin ( $20 \mathrm{mg} /$ day) within 6 and 9 weeks of retinoid administration, respectively. However, because both patients were also given candida immunotherapy (o.2 cc every 2 weeks), it is impossible to accurately evaluate the therapeutic efficacy of isotretinoin in their study.

To the best of our knowledge, the case presented here is the first report of an immunocompetent patient with extensive and recalcitrant common warts that were successfully treated with isotretinoin monotherapy. The response of our patient to this retinoid was impressively rapid: considerable reduction in the number and size of the lesions was already noted after 2 weeks and complete remission after 6 weeks. Another important finding is the lack of any relapse after a 23-month follow-up. The possibility of spontaneous resolution of common warts in our patient cannot be definitely ruled out, but it is extremely unlikely given the protracted course of his disease, the stubborn resistance of the disease to all previous treatments, and the rapid response immediately after the initiation of oral isotretinoin administration. The treatment was well tolerated by our patient, who experienced only minor side effects (cheilitis and dryness of the nasal mucosa with occasional epistaxis).

The exact mechanisms underlying the therapeutic effects of oral isotretinoin in common warts and other cutaneous HPV infections are far from clearly understood. However, this potent and multivalent immunomodulator is known to be capable of inducing apoptosis, down-regulating HPV transcription in affected keratinocytes, and dramatically affecting epithelial differentiation and proliferation (9-11). Because HPV replication is related to the state of keratinocyte differentiation (11), it seems reasonable to assume that isotretinoin may inhibit DNA replication and assembly of HPV, either through its direct action on HPV, or indirectly through modification of epithelial differentiation $(11,12)$.

\section{Conclusions}

Oral isotretinoin monotherapy at a dose of $1 \mathrm{mg} / \mathrm{kg} /$ day can be considered a highly effective and well-tolerated therapeutic modality, which can produce rapid, complete, and long-lasting remission of extensive and recalcitrant common warts in both immunocompetent and immunocompromised patients. 


\section{References}

1. Leto MGP, Santos GF, Jr, Porro AM, Tomimori J. Human papillomavirus infection: etiopathogenesis, molecular biology and clinical manifestations. An Bras Dermatol. 2011;86:306-17.

2. Tsambaos D. A new chapter of dermatotherapy. Derm Beruf Umwelt. 1996;44: 182-3.

3. Monastirli A, Matsouka P, Pasmatzi E, Melachrinou M, Georgiou S, Solomou E, et al. Complete remission of recalcitrant viral warts under oral isotretinoin in a patient with low-grade B-cell lymphoma. Acta Derm Venereol. 2005;85:358-60.

4. Kirnbauer R, Lenz P, Okun M. Human papillomavirus. In: Bolognia JL, Jorizzo JL, Rapini RP, Callen JP, Horn TD, Mancini AJ, editors. Dermatology. 2nd edition. St. Louis, MO: Elsevier; 2008. p. 1183-98.

5. Singh S, Chouhan K, Gupta S. Intralesional immunotherapy with killed Mycobacterium indicus pranii vaccine for the treatment of extensive cutaneous warts. Indian J Dermatol Venereol Leprol. 2014;80:509-14.

6. Katz RA. Isotretinoin treatment of recalcitrant warts in an immunosuppressed man. Arch Dermatol. 1986;122:19-20.

7. Dave DD, Abdelmaksoud A. Low dose isotretinoin as an adjuvant therapy for treatment of different clinical variants of warts: a case series. Dermatol Ther. 2019;32:e12836.
8. Herold M, Nielson C, Longo MI. Isotretinoin and Candida immunotherapy for recalcitrant warts in solid organ transplant recipients. Dermatol Ther. 2019;32: e12803.

9. Tsambaos D, Zimmermann B. Effects of synthetic retinoids on cellular systems. In: Roenigk HJ, Maibach HI, editors. Psoriasis, 2nd ed. New York: Marcel Dekker; 1991. p. 659-707.

10. Bartsch D, Boye B, Baust C, zur Hausen H, Schwarz E. Retinoic acid-mediated repression of human papillomavirus 18 transcription and different ligand regulation of the retinoic acid receptor beta gene in non-tumorigenic and tumorigenic HeLa hybrid cells. EMBO J. 1992;11:2283-91.

11. Tsambaos D, Georgiou S, Monastirli A, Sakkis T, Sagriotis A, Goerz G. Treatment of condylomata acuminata with oral isotretinoin. J Urol. 1997;158:1810-2.

12. Lutzner MA, Blanchet-Bardon C, Puissant A. Oral aromatic retinoid (Ro 10-9359) treatment of two patients suffering with the severe form of epidermodysplasia verruciformis. In: Orfanos CE et al., editors. Retinoids: advances in basic research and therapy. New York: Springer Verlag; 1981. p. 407-10. 